\title{
Sprachförderung im Alltag: Umsetzung in Kindergarten, Kita und Spielgruppe. Videobasierte Fallanalysen
}

\section{Franziska Vogt, Cordula Löffler, Andrea Haid, Nadine Itel, Mandy Schönfelder, Bea Zumwald und Elke Reichmann}

Hohe Erwartungen werden gegenwärtig an verschiedene Initiativen zur Sprachförderung in der frühkindlichen Bildung gestellt. Wirkungsanalysen verweisen auf die Bedeutung der Sprachförderung als Querschnittsaufgabe und damit auf die Sprachförderkompetenz der Fachkräfte, Kinder in ihrem Spracherwerb adaptiv zu unterstützen. Die hier diskutierte Interventionsstudie umfasst eine Weiterbildung für frühpädagogische Fachkräfte aus Spielgruppe, Kita und Kindergarten in Deutschland und der Schweiz, die fünf ausgewählte Strategien der Sprachförderung im Alltag thematisiert. Es werden die Strategien und ihre Umsetzung durch die Fachkräfte anhand von Fallstudien auf der Basis der Videoanalyse aufgezeigt.

\section{Einleitung: Sprachförderung in der frühkindlichen Bildung}

Sprachliche Kompetenzen sind für den weiteren Bildungsverlauf von Kindern entscheidend (Dickinson \& Porche, 2011; Wasik \& Hindman, 2011). Die Erwartungen an den Nutzen von Sprachförderung in der frühkindlichen Bildung für Kinder bildungsferner Familien und Familien mit Migrationshintergrund sind sehr hoch (Rossbach \& Hasselhorn, 2012). Zahlreiche und sehr unterschiedliche Sprachförderprojekte in diversen Kontexten werden aktuell realisiert (für einen Überblick zu den Projekten für die Schweiz: Hutterli \& Vogt, 2014; für Deutschland: Lisker, 2011). Diese Initiativen richten sich an die institutionellen Kontexte der frühkindlichen Bildung und Betreuung wie Kindergarten, Kita und Spielgruppe.

Wirkungsstudien zu Initiativen zur Sprachförderung zeigen unterschiedliche Ergebnisse, da einige methodische Herausforderungen und Probleme mit der Übertragbarkeit auf andere Kontexte bestehen (Kuger, Sechtig \& Anders, 2012). Insgesamt werden die pädagogische Qualität sowie die professionellen Kompetenzen der frühpädagogischen Fachkräfte als zentral eingeschätzt (Anders, 2012). Für die Implementation der Sprachförderung ist die professionelle Kompetenz 
der pädagogischen Fachkräfte entscheidend (Kucharz, Uemminghaus, Gasteiger-Klicpera \& Knapp, 2009). Diese Sprachförderkompetenz umfasst nach Fried (2008a) ein «Ensemble aus Motiv-, Wissens- und Könnenskonstituenten» (S. 266). Frieds Untersuchungen zeigen, dass die frühpädagogischen Fachkräfte im Bereich der Beziehungsqualität generell ein hohes Niveau erreichen, es jedoch an Strategien für die Initiierung und die Nutzung von pädagogischen Alltagsmomenten für «sozialkonstruktivistische Bildungssituationen» fehlt (Fried, 2008b, S. 148). Die frühpädagogische Fachkraft soll in einer konkreten Situation die Interaktion mit einem oder mehreren Kindern so gestalten, dass die Sprachförderung dem Sprachentwicklungsstand des Kindes angepasst ist und in der entwicklungsproximalen Zone liegt. Die Bedeutung der Adaptivität der pädagogischen Fachkraft als Teil der professionellen Kompetenz wurde in Untersuchungen aus der (Schul-)Pädagogik und aus der Spracherwerbsforschung postuliert (Beck et al., 2008; Buschmann, 2009; Dannenbauer, 2002; Vogt, 2013; Ritterfeld, 2000). Die professionelle Kompetenz für Sprachförderung lässt sich durch Weiterbildung positiv beeinflussen (Buschmann \& Jooss, 2011; Girolametto, Weitzmann, Lefebvre \& Greenberg, 2007). Der Grad der Professionalisierung ist in den verschiedenen institutionellen Kontexten in der Schweiz und in Deutschland sehr unterschiedlich und reicht beispielsweise von einer Weiterbildung für die Spielgruppe zur Berufsausbildung für Erziehende und zum Hochschulstudium für Kindergartenlehrpersonen in der Schweiz. Kontrovers diskutiert wird die Art der Sprachförderung in der frühkindlichen Bildung, speziell die Frage, ob eine breit angelegte, im Alltag verankerte Sprachförderung oder spezifische Trainingsprogramme wirkungsvoller seien. Während einzelne Studien für spezifische Programme Wirkung ausweisen (Jäger et al., 2012), zeigen mehrere Evaluationen keine Wirkungen (Gasteiger-Klicpera, Knapp \& Kucharz, 2010; Gretsch \& Fröhlich-Gildhoff, 2012; Sachse, Budde, Rinker \& Groth, 2012; Schöler \& Roos, 2011). Hingegen bieten die Interaktionen im Alltag zahlreiche Möglichkeiten zur Sprachförderung. «Wie beim natürlichen Erstspracherwerb soll das Kind in einer alltagsähnlichen Kommunikationssituation aus dem Input intuitiv Sprachregeln ableiten, diese erproben und korrigieren, falls sich seine Vermutungen über die neue Sprachregel als falsch oder ungenau erweisen" (Suchodoletz, 2013, S. 126). Um diese Möglichkeiten zu nutzen, ist eine hohe professionelle Kompetenz nötig.

Die Professionalisierung ist möglicherweise eine Bedingung für die Wirksamkeit der Sprachförderung, denn sie erlaubt einerseits, dass die Förderung alltagsimmanent geschieht, und andererseits, dass die Sprachförderung an den individuellen Entwicklungsstand des Kindes angepasst ist. Für die Untersuchung der professionellen Sprachförderkompetenz wurden für die vorliegende Studie videografierte Handlungssituationen in verschiedenen Kontexten der frühkindlichen Bildung und Betreuung systematisch analysiert. ${ }^{1}$ Dazu wurde ein Kategoriensystem entwickelt, mit dem die Sprachförderkompetenz der frühpädagogischen Fachkräfte in realen alltäglichen pädagogischen Situationen erfasst werden 
soll. Damit soll untersucht werden, wie sich die Sprachförderstrategien in den Interaktionen der Fachkräfte mit den Kindern in verschiedenen Kontexten der frühkindlichen Bildung und Betreuung zeigen und wie sie mit einem Kategoriensystem für die Videoanalyse systematisch erfasst werden können.

\section{Strategien für die Sprachförderung im Alltag}

Für die Sprachförderung im Alltag werden Strategien ausgewählt und konkretisiert, die das Initiieren und Nutzen von Bildungsmomenten in Interaktionen ermöglichen: Dialog mit Kindern, Fragen, Wortschatz, Modellieren und Redirect. Diese Strategien sollen in unterschiedlichen Kontexten im Alltag angewandt und dem Entwicklungsstand der Kinder im Spracherwerb angepasst werden können.

\section{Im Dialog mit Kindern}

Längere dialogische Interaktionen zwischen der frühpädagogischen Fachkraft und dem Kind, bei denen die Fachkraft die Aktivitäten, Äusserungen und Interessen des Kindes wahrnimmt und ihm eigene Gesprächsbeiträge ermöglicht, sind ein bestimmendes Qualitätsmerkmal frühkindlicher Bildung. SirajBlatchford und Sylva (2004) prägten dafür den Ausdruck sustained shared thinking. Im deutschen Sprachraum wurde das Konzept von König (2007) mittels einer Videoanalyse in Kitas untersucht und mit dem Begriff «bewusst dialogisch-entwickelnde Denkprozesse» umschrieben, sowie von Hopf (2012) auf das naturwissenschaftliche Denken in der Elementarpädagogik adaptiert. Die Dialoge sollen inhaltlich auf ein Thema fokussieren und zumindest die Form A-B-A-B haben, also zwei Äusserungen eines Kindes im Wechsel mit zwei Äusserungen einer pädagogischen Fachperson (König, 2009). Für die Beiträge der Fachkraft zum Dialog wird scaffolding, extending, discussing, modelling und playing unterschieden (Siraj-Blatchford, Sylva, Muttock \& Bell, 2002). Auch die Handlungen eines Kindes können Anknüpfungspunkt für sprachfördernde Dialoge sein. Die Fachkraft verbalisiert, was das Kind macht, sie reagiert spielerisch und handelnd auf das Kind und versprachlicht die eigenen Handlungen (Albers, 2009; Best, Laier, Jampert, Sens \& Leuckefeld, 2011; Motsch, 2010).

\section{Sprachfördernde Fragen}

Die Frage als pädagogisches Handlungsmuster (Briedigkeit, 2011) und sprachdidaktisches Werkzeug im institutionellen Rahmen organisiert sehr häufig die Fachkraft-Kind-Interaktion. Es besteht dabei ein enger Zusammenhang zwischen der Art der Frage und wie sich Kinder sprachlich in den Dialog einbinden können (König, 2009). Die drei Grundtypen der Frage, Entscheidungs-, Alternativ- und Ergänzungsfragen (Linke, Nussbaumer \& Portmann, 2004), treten antwortbezogen in Wechselbeziehung zum Spracherwerbsprozess 
- ein Illokutionspotenzial im Frage-Antwort-Dialog (Meibauer, 2008). Die Entscheidungsfrage mit Prädikaterststellung zielt in ihrer Struktur auf die Zustimmung oder Ablehnung einer Äusserung mit den Antwortpronomen «Ja" und «Nein». Dieses Frageformat begleitet einen impliziten Lernprozess (Sazgun, 2006), indem die kindlichen Sprechenden über die Imitation einer Modelläußerung zur eigenständigen Konstruktion gelangen. Bei der Alternativfrage werden Sprachstrukturen als Antwortmöglichkeiten von der Fachkraft modellhaft vorgegeben und können vom Kind in der Antwort ausgewählt und produziert werden. Mithilfe dieser Wahlstruktur können alle Sprachebenen fördernd und unterstützend aufgegriffen werden. Die Ergänzungsfrage (,W'-Frage) als ein weiteres Frageformat fordert die höchste Komplexität in der Antwortstruktur. Indem eine syntaktische Ergänzung (Nominal-, Präpositionalphrase oder Nebensatzstruktur) hervorgerufen wird, kann diese Frage den Wortschatzaufbau und Grammatikerwerb begleiten. Für die Sprachförderung wird häufig der Mehrwert offener Fragen postuliert (Briedigkeit, 2011; King et al., 2011; Siraj-Blatchford \& Manni, 2008). Die Prozessqualität von Fragen kennzeichnet sich jedoch durch eine Anpassung an das sprachliche Vermögen des Kindes, indem die Fragestrategie entwicklungsproximal (Dannenbauer, 1994) und sukzessiv aufbauend eingesetzt wird.

\section{Wortschatz}

Ein grosser Wortschatz wird als ein wichtiger Prädiktor für die schulische Laufbahn angesehen (Dickinson \& Porche, 2011; Szagun, Steinbrink, Franik \& Stumper, 2006). Natürliche Interaktionen, die in Spielanlässen und Aktivitäten in den Einrichtungen auftreten, bieten eine grosse Vielfalt an Möglichkeiten für Wortschatzinput. Dabei spielen zum einen die Quantität des Sprachinputs (Huttenlocher, Vasilyeva, Waterfall \& Vevea, 2007) und zum anderen ein reichhaltiges und abwechslungsreiches Wortschatzangebot (Torr \& Scott, 2006) eine wichtige Rolle für die Aufnahme, Verarbeitung und Speicherung neuer Wörter. Zudem sind sprachlernunterstützende Strategien nötig, die es dem Kind erleichtern, den Sprachinput aufzunehmen und zu verarbeiten (Lever \& Sénéchal, 2011; Wasik \& Hindman, 2011). Dazu gehören Wortpräsentation, Worterarbeitung und Wortfestigung. Damit ein Kind auf ein neues Wort aufmerksam wird und die Lautstruktur wahrnehmen kann, sollte es von der frühpädagogischen Fachkraft deutlich, langsam und bewusst artikuliert präsentiert werden. Bei der Worterarbeitung wird die Bedeutung geklärt, indem beispielsweise auf das entsprechende Objekt oder auf ein Bild verwiesen, das Wort mit Gestik und Mimik veranschaulicht oder das Wort erklärt wird. Zur Festigung eines neuen Wortes sind die mehrmalige Wiederholung, die Vernetzung mit andern Wörtern und die Verknüpfung mit der Lebenswelt des Kindes fördernd.

\section{Model lieren}

Die Modellierungstechniken sind spracherwerbsförderliche Verhaltensweisen, die vor allem in der intuitiven Didaktik von Eltern mit ihren Kleinkindern 
beobachtet werden können. Sie unterstützen das Kind in seinem Spracherwerb nachweislich, indem die erwachsene Person das Kind adaptiv im Dialog korrigiert, indem sie eine korrekte Satzstruktur oder eine inhaltliche Erweiterung anbietet (Dannenbauer, 2002; Motsch, 2010).

Es werden hierbei vier Modellierungstechniken (Motsch, 2010), die einer kindlichen Äusserung folgen, unterschieden. Im korrektiven Feedback wird eine fehlerhafte Äusserung des Kindes reaktiv aufgenommen und in korrigierter Form wiedergegeben. Die Extension umfasst eine Erweiterung auf der Inhaltsebene, die Expansion hingegen meint eine Ergänzung bzw. Vervollständigung des Satzes auf syntaktischer Ebene. Die Umformung beinhaltet die morphosyntaktische Abwandlung des kindlichen Sprachmaterials.

\section{Redirect}

Auch die anderen Kinder in der Gruppe sind für den Spracherwerb von Bedeutung (Albers, 2009; Licandro \& Lüdtke, 2013). Für Kinder mit sprachlichen Schwierigkeiten (Migrationshintergrund oder Sprachentwicklungsstörung) kann die Aufnahme und Aufrechterhaltung von Peer-Interaktionen jedoch eine Herausforderung darstellen; sie initiieren seltener Peer-Kontakte. Um sie in der Peerinteraktion zu unterstützen, wurde die Sprachfördertechnik Redirect entwickelt, bei der eine Anfrage eines Kindes an die Fachkraft durch diese an ein anderes Kind weitergeleitet wird (Schuele, Rice \& Wilcox, 1995). Im Idealfall wird dieser Impuls vom Kind aufgegriffen und eine Kind-Kind-Interaktion initiiert (Albers, 2009). Es gibt verschiedene Strategien der Weiterleitung: Beim Modell werden dem Kind genaue Formulierungen vorgegeben, die das Kind eins-zu-eins übernehmen kann. Beim Vorschlag erhält es lediglich den Impuls, sich an ein anderes Kind zu wenden und muss die Formulierung seines Anliegens selbst finden. Der Hinweis ist die anspruchsvollste Form des Redirects, da die Möglichkeit zur Interaktion mit einem anderen Kind von der Fachkraft lediglich angedeutet wird (Schuele, Rice \& Wilcox, 1995).

\section{Methode}

Das Forschungsprojekt Sprachförderung im Alltag von Kindergarten, Kita und Spielgruppe ist eine Interventionsstudie zur Förderung der professionellen Kompetenzen im Bereich Sprachförderung, welches von der Internationalen Bodensee Hochschule unterstützt wurde. Ziel ist es, die Sprachförderkompetenz der Fachkräfte zu erweitern. Vor und nach der Intervention wurde die Sprachförderkompetenz der frühpädagogischen Fachkräfte mit Fragebogen und Filmvignetten (Itel, 2012) sowie mit Videobeobachtung und Videorecall im realen Kontext des Kindergartens, der Kita bzw. der Spielgruppe untersucht. Auf Grund der begrenzten Ressourcen musste auf ein experimentelles Design mit einer Kontrollgruppe sowie auf eine Wirkungsanalyse auf der Ebene der Kinder verzichtet werden. 
Die Stichprobe umfasst insgesamt 45 Frühpädagoginnen mit 11 Kindergartenlehrpersonen und 22 Spielgruppenleiterinnen aus der Schweiz sowie 12 Erzieherinnen in Kitas aus Deutschland. Für diesen Beitrag wurden drei Frühpädagoginnen ausgewählt, die durch ihre Organisationsformen und Lernarrangements für eine Mehrheit der jeweiligen Kontexte repräsentativ sind. Die drei ausgewählten Frühpädagoginnen unterscheiden sich in Bezug auf die Selbsteinschätzung ihrer Sprachförderkompetenz im Fragebogen sowie in Bezug auf Alter, Berufserfahrung und Weiterbildung nicht signifikant von der Gesamtgruppe. Nur die ausgewählte Spielgruppenleiterin ist mit mehr als 10 Jahren Berufserfahrung erfahrener als die meisten anderen Spielgruppenleiterinnen.

Die Intervention umfasste eine gemeinsame Fortbildung. In einem ersten Teil wurden in zwei Tagen Grundlagen zu Spracherwerb und Sprachförderung sowie Trainings zu jeder der fünf Strategien angeboten. Zur Vertiefung wurde nach zwei Monaten an einem Begleitnachmittag in kleineren Gruppen die Umsetzung besprochen. Im Anschluss an den Begleitnachmittag wurden die Frühpädagoginnen zudem mit einer wöchentlichen Erinnerungsmail und einem Tischkalender mit Merksätzen (siehe Tab. 1) dazu angeregt, sich wöchentlich eine der Strategien zur Umsetzung vorzunehmen.

\section{Tabelle 1: Merksätze zur Umsetzung der Sprachförderung im Alltag}

\begin{tabular}{|l|l|}
\hline Strategie & Merksätze des Tischkalenders \\
\hline Im Dialog mit Kindern & $\begin{array}{l}\text { Ich nehme wahr, was das Kind macht, und versprachliche seine } \\
\text { Handlung. } \\
\text { Ich nehme das Thema des Kindes wahr und trete mit ihm in einen } \\
\text { längeren Dialog. }\end{array}$ \\
\hline Sprachfördernde Fragen & $\begin{array}{l}\text { Für gemeinsame Gespräche plane ich Fragen sorgfältig. } \\
\text { Ich formuliere geschlossene, geöffnete und offene Fragen, um alle Kinder } \\
\text { sprachlich zu erreichen. }\end{array}$ \\
\hline Wortschatz & $\begin{array}{l}\text { Ich wähle zwei bis drei neue Wörter zu einer Bilderbuchgeschichte aus } \\
\text { und verwende diese Wörter bewusst viele Male. }\end{array}$ \\
\hline Modellierungstechniken & $\begin{array}{l}\text { Ich signalisiere dem Kind, dass ich es inhaltlich verstanden habe, und } \\
\text { wiederhole die Aussage des Kindes in korrekter oder erweiterter Form. }\end{array}$ \\
\hline Redirect & $\begin{array}{l}\text { Ich schlage dem Kind vor, ein anderes Kind anzusprechen. } \\
\text { Ich sage dem Kind, was es zu dem anderen Kind sagen kann. }\end{array}$ \\
\hline
\end{tabular}

Für die Videoanalyse wurde das gesamte videografierte Material, pro Frühpädagogin zwischen anderthalb und drei Stunden je Testzeitpunkt, in einer Grobstrukturierung gesichtet. Dabei wurden die didaktische und organisatorische Form festgehalten sowie die Interaktionen zwischen Frühpädagogin und Kind von mindestens vier Turns markiert. Auf Grund der Grobstrukturierung wurden drei Sequenzen von je 15 Minuten Dauer ausgewählt, die eine hohe Dichte an Eins-zu-eins-Interaktionen der Frühpädagogin mit einem Kind aufwiesen, darunter mindestens eine Sequenz mit einem Bilderbuch und einer 
unstrukturierten Phase wie Freispiel oder Essenssituation. Diese 45 Minuten pro Frühpädagogin wurden mit Hilfe des deduktiv entwickelten Kategoriensystems zu den Sprachförderstrategien (Tab. 2) vertieft analysiert. Alle Sprachförderstrategien wurden einzeln fokussiert und kodiert. Dadurch wurden auch die Zeitausschnitte des Eventsampling (Reis, \& Gable 2000) für jede Strategie unabhängig festgelegt. So kann beispielsweise die Fragestrategie überlappend in einer Sequenz eines länger andauernden Dialogs vorkommen, es werden jedoch auch einzelne Fragen ohne sustained shared thinking kodiert. Für die Intercoder-Reliabilität in Bezug auf die Kategorien und das Eventsampling wurde eine Übereinstimmung von über $70 \%$ erreicht.

\section{Tabelle 2: Kategoriensystem für die Videoanalyse zur Sprachförderung im Alltag}

\begin{tabular}{|c|c|c|}
\hline \multicolumn{3}{|l|}{ Im Dialog mit Kindern } \\
\hline Einstieg & \multicolumn{2}{|l|}{$\begin{array}{l}\text { Erwachsenen-initiiert } \\
\text { Kind-initiiert }\end{array}$} \\
\hline Anzahl Beteiligte & \multicolumn{2}{|l|}{$\begin{array}{l}\text { Dyadisch } \\
\text { Polyadisch }\end{array}$} \\
\hline $\begin{array}{l}\text { Beitrag pädagogische } \\
\text { Fachkraft }\end{array}$ & \multicolumn{2}{|l|}{$\begin{array}{l}\text { Scaffolding } \\
\text { Extending } \\
\text { Discussing } \\
\text { Modelling } \\
\text { Playing }\end{array}$} \\
\hline Beitrag Kind & \multicolumn{2}{|l|}{$\begin{array}{l}\text { Playing } \\
\text { Discussing } \\
\text { Extending } \\
\text { Spricht anderes Kind an }\end{array}$} \\
\hline Verbalisierung & \multicolumn{2}{|c|}{$\begin{array}{l}\text { Verbalisieren Handlung Kind } \\
\text { Verbalisieren eigene Handlung } \\
\text { Verbalisieren eigene Handlung mit Begründung }\end{array}$} \\
\hline \multicolumn{3}{|l|}{ Fragen } \\
\hline \multirow{6}{*}{$\begin{array}{l}\text { Fragestruktur der } \\
\text { Fachkraft }\end{array}$} & Prädikaterststellung & \\
\hline & Alternativfrage & \\
\hline & Interrogativpronomen & $\begin{array}{l}\text { IP (Nominativ) } \\
\text { IP (Kasusmarkierung) } \\
\text { IP (Prädikat) } \\
\text { IP (Nebensatz) }\end{array}$ \\
\hline & Interrogative Adverbien & $\begin{array}{l}\text { W-Wort } \\
\text { W-Wort mit Präposition } \\
\text { Präpositionaleinstieg mit w-Wort }\end{array}$ \\
\hline & Interrogative Adjektive & \\
\hline & Interrogativartikel & \\
\hline $\begin{array}{l}\text { Antwortverhalten des } \\
\text { Kindes }\end{array}$ & A unklar & \\
\hline
\end{tabular}




\begin{tabular}{|c|c|c|}
\hline & $\begin{array}{l}\text { fragenadäquate Antwort } \\
\text { (expressiv) }\end{array}$ & $\begin{array}{l}\text { eigener (erweiternder) Input } \\
\text { fehlerhaft/unvollständig } \\
\text { korrekt }\end{array}$ \\
\hline & $\begin{array}{l}\text { frageninadäquate Antwort } \\
\text { (expressiv unpassend) }\end{array}$ & \\
\hline & $\begin{array}{l}\text { unverständliche Antwort } \\
\text { (mangelnde Tonqualität) }\end{array}$ & \\
\hline & nonverbale Reaktion & \\
\hline & keine Reaktion & \\
\hline \multicolumn{3}{|l|}{ Wortschatz } \\
\hline anbieten & $\begin{array}{l}\text { spezifischer Begriff } \\
\text { andere Wortart } \\
\text { Wortpräsentation }\end{array}$ & \\
\hline erarbeiten & $\begin{array}{l}\text { veranschaulichen } \\
\text { Mimik Gestik } \\
\text { Merkmalsbeschreibung }\end{array}$ & \\
\hline festigen & $\begin{array}{l}\text { Repetition } \\
\text { vertiefen } \\
\text { Lebensweltbezug }\end{array}$ & \\
\hline \multicolumn{3}{|l|}{ Modellierungstechniken } \\
\hline \multirow{4}{*}{$\begin{array}{l}\text { Angemessene Reaktion } \\
\text { auf kindliche Äusserung }\end{array}$} & Extension (semantisch) & \\
\hline & Expansion (syntaktisch) & \\
\hline & Umformung & $\begin{array}{l}\text { Reine Umformung } \\
\text { Umformung + Extension } \\
\text { Umformung + Expansion }\end{array}$ \\
\hline & Korrektives Feedback & $\begin{array}{l}\text { Semantisch-lexikalisch } \\
\text { Morpho-syntaktisch } \\
\text { Phonetisch-phonologisch }\end{array}$ \\
\hline $\begin{array}{l}\text { Unangemessene } \\
\text { Reaktion }\end{array}$ & $\begin{array}{l}\text { Wiederholung } \\
\text { explizite/direkte Korrektur } \\
\text { fehlerhafte Modellierung } \\
\text { nachsprechen lassen }\end{array}$ & \\
\hline \multicolumn{3}{|l|}{ Redirect } \\
\hline Art des Redirects & $\begin{array}{l}\text { Modell } \\
\text { Vorschlag } \\
\text { Hinweis }\end{array}$ & \\
\hline Initiierung & $\begin{array}{l}\text { Kind } \\
\text { Erwachsener }\end{array}$ & \\
\hline Aufnahme des Redirects & $\begin{array}{l}\text { erfolgreich } \\
\text { nicht erfolgreich }\end{array}$ & erneuter Impuls \\
\hline $\begin{array}{l}\text { Gestaltung der } \\
\text { Interaktion }\end{array}$ & $\begin{array}{l}\text { selbstständig } \\
\text { erwachsenengestützt }\end{array}$ & \\
\hline
\end{tabular}




\section{Ergebnisse}

Die drei für die Fallstudien ausgewählten Frühpädagoginnen verwenden didaktische Settings, die in den jeweiligen Institutionen (Spielgruppe, Kita und Kindergarten) für die Mehrheit der Frühpädagoginnen des Forschungsprojektes repräsentativ sind. Es wird zuerst ein Fallbeispiel einer Schweizer Spielgruppe, danach einer deutschen Kita und abschliessend eines Schweizer Kindergartens dargestellt. In der Analyse der Videos wurde das didaktische Setting ebenfalls erfasst: in allen drei institutionellen Kontexten gelingen längere Dialoge zwischen den Frühpädagoginnen und den Kindern sehr häufig im didaktischen Setting des freien Spiels und beim Betrachten und Vorlesen eines Bilderbuchs. Deshalb werden je ein Fallbeispiel zum freien Spiel und zur Bilderbuchbetrachtung ausgewählt. Im Gegensatz zur Spielgruppe und den Kitas in Deutschland kommen in Schweizer Kindergärten zusätzlich stärker geführte Sequenzen mit schulähnlicher Aufgabenstruktur vor. Das dritte Fallbeispiel zeigt somit eine solche geführte Sequenz. Mit Hilfe dieser drei unterschiedlichen Fallbeispiele aus verschiedenen Kontexten und didaktischen Settings soll gezeigt werden, inwiefern die Strategien für die alltagsintegrierte Sprachförderung tatsächlich in verschiedenen Settings angewendet werden können.

\section{Fallstudie 1: Sprachförderung in einer spielgruppe in der Schweiz}

Die Spielgruppenleiterin hat anhand eines Bilderbuchs mit Figuren und Gegenständen eine Geschichte erzählt, bei der es darum ging, welches Tier welche Nahrung mag. In der freien Spielphase holen die Kinder eine Schachtel mit Tierfiguren. K1 und K2, wie auch alle anderen Kinder der Spielgruppe, haben nur sehr geringe Deutschkenntnisse. Das Gespräch ist in Schweizer Dialekt geführt, für die Nachvollziehbarkeit werden die Aussagen zusätzlich in Standardsprache wiedergegeben.

1. $<\mathrm{K} 1$ nimmt ein Schaf und stellt es auf den Rücken des Nashorns, lacht und schaut die Frühpädagogin an>

2. FP: Das isch aber es muetigs Tier do, he, grad ufs grossi ufe stiegts. (Das ist aber ein mutiges Tier hier, es steigt gleich auf das grosse rauf) (...)

3. K2: Da isch alle Tiere. (Das ist alle Tiere)

4. FP: Da sind alli Tier. (Das sind alle Tiere)

5. K2: Jo. (ja)

6. FP: Und weles isch denn e gföhrlichs Tier? (-) Weles Tier isch denn do gefährlich? Isch das gefährlich $\{\mathrm{D}\}$ ? (Und welches ist denn ein gefährliches Tier? Welches Tier ist denn hier gefährlich? Ist das gefährlich?) <zeigt auf ein Tier>

7. K2: Jo. (ja)

8. FP: Das isch gefährlich. (Das ist gefährlich)

9. K2: De do. <hebt Tier hoch> (Dieser da) 
10. FP: Warum isch de gefährlich? (Warum ist das gefährlich?)

11. $<\mathrm{K} 2$ rammt das Nashorn mit dem Horn voran in einen dort stehenden Plastikkübel hinein>

12. FP: Wa het denn de döte? <tippt auf die Nase> Was isch do druf, uf de Nase? (Was hat es denn da? Was ist hier drauf, auf der Nase?)

13. K2: Weiss nöd. (Weiss nicht)

14. FP: Es Horn.(Ein Horn)

15. K1: Horn.

16. FP: Es Horn. Drum heisst's N-a-shorn. Wil er do e Horn druf het do, oder. (Ein Horn, darum heisst es Nashorn, weil er da ein Horn darauf hat, oder.)

Das Kind K1 initiiert den Dialog nonverbal, indem es die Aufmerksamkeit der Frühpädagogin für ihre Handlungen sichert, sie macht jedoch keinen eigenständigen sprachlichen Beitrag. Die Spielgruppenleiterin nimmt wahr, welche Handlung das Kind ausführt und versprachlicht diese (Verbalisierung Handlung Kind). Die Sprachförderstrategie des Verbalisierens kann als eine adaptive Form der Anbahnung von sustained shared thinking bei Kindern mit sehr geringen Deutschkenntnissen gesehen werden. Der Dialog zwischen K2 und der Frühpädagogin wurde als sustained shared thinking kategorisiert. K2 gibt eigenständige sprachliche Äusserungen ein (Zeile 3, 9) und es erweitert den Dialog mit playing in Zeile 11.

In Bezug auf Fragen zeigt sich ein Gesprächsbeitrag der Frühpädagogin mit drei aufeinanderfolgenden Fragen, zwei Ergänzungsfragen und einer Entscheidungsfrage, die den Turn abschliesst (Zeile 8). An diesem Beispiel kann skizziert werden, dass Fragen dem Phänomen der Mündlichkeit und deren Regeln unterliegen, mit prosodischen Elementen wie Akzent und Pausensetzung die Übertragung des Rederechts markierend. Die Fragekette wechselt von der anspruchsvolleren Frage, die nach einer kurzen Pause vom Kind nicht beantwortet wird, zur letztgenannten einfacheren Frage, deren Beantwortung verbindlich an das Kind delegiert wird. Die Benennung eines Tiermerkmals wird hingegen in Form einer Ergänzungsfrage mit,$w^{`}$-Wort evoziert (Zeile 12).

Im Bereich Wortschatz bietet die Spielgruppenleiterin den Kindern den spezifischen Begriff des Horns sowie das Wort "gefährlich" (andere Wortart) an. Zuerst erarbeitet und veranschaulicht sie den Begriff Horn mit Gestik (Zeile 12), indem sie auf die Nase tippt und mit den Händen das Horn gestisch darstellt. Anschliessend erklärt sie inmitten einer Merkmalsbeschreibung, dass das Nashorn ein Horn hat (Zeile 14), und sie wiederholt den Begriff mehrmals (festigen, Repetition)

Als Modellierungstechnik verwendet sie in Zeile 4 ein korrektives Feedback auf der morphosyntaktischen Ebene (K2: Da isch alle Tiere. FP: Da sind alli Tier). Das Beispiel zeigt sehr gut das Potenzial korrektiven Feedbacks. Das Gespräch wird nicht unterbrochen, im Gegenteil, das korrektive Feedback bestätigt das Kind in seiner Äusserung inhaltlich. 
Redirect kommt in dieser Sequenz nicht vor, es wird hier eine andere Stelle aus der Videoanalyse dieser Spielgruppenleiterin angeführt. Die Kinder und die Frühpädagogin sitzen rund um einen Tisch:

1. FP: [Name K] wenn du nöd wötsch geh den seisch «Ich will nüme». Gell? Lueg, es het jede sin eigene Znüni. (Name K], wenn du nicht geben willst, dann sagst du es: «Ich will nicht mehr». Gell? Schau, jeder hat sein eigenes Frühstück.)

2. K: Wötts nöd geh. (Will es nicht geben.)

3. FP: Jo genau denn seisch es. «Ich will nüme geh». (Ja genau dann sagst du es: «Ich will nicht mehr geben».)

Die Spielgruppenleiterin verwendet Redirect als ein Modell, sie modelliert den Satz, den das Kind an ein anderes Kind adressieren kann. Das Kind greift das Redirect erfolgreich auf und die Spielgruppenleiterin wiederholt das Modell noch einmal zur Bestärkung.

Fallstudie 2: Sprachförderung einer Erzieherin in einer Kita in Deutschland

Die Erzieherin liest einer Gruppe von drei Kindern ein Buch vor, sie sitzen alle auf dem Sofa. Dabei entsteht das folgende Gespräch:

1. K3: Ich ha mol () und am Mittwoch, da hab ich einen toten Igel auf der Strasse gesehn.

2. FP: Oooh. Hat ma den überfahre? Wahrscheinlich, oder?

3. K3: Leider.

4. FP: Oooh. Ja manchmal sieht man hin und wieder ein Tierle auf der Strasse überfahre, gell.

5. K3: So wie das Eichhörnli do hane.

6. FP: Ja. Und weisch was i heut Morgen gseh han?

7. K3: Mhm.

8. FP: En Dachs. Weisch wasn Dachs isch?

9. K3: Mhm.

10. FP: Der war auch überfahre. Das isch e ganz ganz grosses Tier. <zeigt gestisch die Grösse des Tieres> Bini gad bissle erschrocke wo das mitten auf der Strasse glege isch. $\ldots$

11. K4: // ich hab ein Fuchs gsehn.

12. FP: En Fuchs hend ihr scho mol gsehe.

13. K4: En tote.

14. FP: Au, hmmm

15. K1 und K2 im Chor: Und, und ich hab schon mal gesehn wo wo über die Strasse glaufen ist.

16. FP: echt war da, wars da, war es da hell am Tag oder war es da dunkel?

17. K1 und K2 im Chor: hell. 
18. FP: Echt? Weil normalerweise mögen die Füchse oder gehen die Füchse eher abends, gell, weil es dann ein bissl dämmert.

19. K3: Weil, weil da sind koni Jäger meh.

20. FP: Das könnt vielleicht ein Grund sein, ja.

In Bezug auf die Strategie, im Dialog mit Kindern' entwickelt sich hier ein längeres, polyadisches Gespräch des sustained shared thinking zu einem vom Kind initiierten Thema. Die Frühpädagogin unterbricht das Vorlesen des Bilderbuchs, vertieft und trägt neue Aspekte bei (Zeile 6; Zeile 16, 18). Auch die Kinder bringen weitere Aspekte ein, beispielsweise die Überlegung, dass die Füchse am Abend rauskommen, weil die Jäger dann nicht mehr jagen. Für die Erzieherin wie für die Kinder wurden die Codes discussing und extending vergeben.

In Zeile 2 erfragt die pädagogische Fachkraft eine Vermutung (Oooh. Hat ma den überfahre? Wahrscheinlich, oder?), die scheinbar auf Zustimmung oder Ablehnung zielt. Diese Form der Frage (Vergewisserungsfrage) hat eine kommunikativ-pragmatische Funktion, sodass sie nicht als obligatorische Fragestellung aufscheint. Das Kind erweitert den Gesprächsstrang um eine situationswertende Aussage (leider). In Zeile 6 und 8 finden sich die generell häufigen Entscheidungsfragen mit Prädikaterststellung (IP Prädikat). Die Frühpädagoginnen wurden im Rahmen der Fortbildung auf das Potenzial von Alternativfragen als Sprachmodell aufmerksam gemacht. In Zeile 19 bedient die Erzieherin diese Strategie: ,war es da hell am Tag oder war es da dunkel?' und ermöglicht den Kindern, auf eine vorgegebene komplexe Struktur zurückzugreifen und diese sprachlich zu reproduzieren. Diese Variation erweist sich als adaptiv, K1 und K2 konnten sich stärker einbringen, als dies zu Beginn des Gesprächs möglich war. Zugleich ist das Thema der nachtaktiven Tiere angepasst an die Zone der nächsten Entwicklung für K3, das nun mit einem weiterführenden Gedanken am Gesprächsverlauf teilnehmen kann.

Für die Strategie Wortschatz bietet die Frühpädagogin in diesem Gespräch das Wort ,Dachs" an, ein spezifischer Begriff, den sie in Zeile 8 einführt und wiederholt (festigen, Repetition), zudem werden die Merkmale des Dachses beschrieben (Merkmalsbeschreibung.) Sie ergänzt dies mit einer Geste, die die ungefähre Grösse eines Dachses zeigt. Das Wort gross' in Zeile 10 wurde als weiterer Begriff der Wortschatzförderung markiert, da es sich um ein Adjektiv (andere Wortart) handelt, das sie bewusst mit Gestik hervorhebt und zeigt.

Für die Strategie der Modellierung findet sich hier ein Beispiel einer Umformung. Da die kindlichen Äusserungen keine Korrektur erfordern, modelliert die FP vergleichsweise wenig. In Zeile 12 bietet sie eine sprachliche Anregung durch eine Umformung + Extension in den Verbformen, sodass dem Kind eine neue morphosyntaktische Struktur präsentiert wird. 
Fallstudie 3: Sprachförderung im Kindergarten in der Schweiz

Die Kinder haben in Dreiergruppen Bilder des Backablaufs auf Streifen geklebt und bekommen nun den Auftrag, das Vorgehen beim Backen reihum zu erzählen. Die Kindergärtnerin geht von Gruppe zu Gruppe und lässt sich den Ablauf anhand der Bilder erzählen:

1. FP: hmm aso jetzt hämmer Salz, Mehl, Schoggi, Eier, Bachpulver, was chönnt echt da no si? Weisch das [Name K1]? (Also jetzt haben wir Salz, Mehl, Schokolade, Eier, Backpulver, was könnte wohl das noch sein? Weisst du das [Name K1]?

2. $\mathrm{K} 1 \mathrm{mmh}$

3. FP: wer chan em [Name K1] hälfe? (Wer kann [Name K1] helfen?)

4. K2: Zucker (...)

5. D'[Name K2] häts gseit vorher gseit, söll si's nomol säge chasch sie nomol froge. ([Name K2] hat es vorher gesagt. Soll sie es nochmals sagen, kannst sie nochmal fragen)

6. K1: weiss es nüme (weiss es nicht mehr)

7. FP: weisch es nüme, söll si's nomol säge? (weisst es nicht mehr, soll sie es nochmals sagen?)

8. K1: ja

Dies ist eine erarbeitende Sequenz, in der die Kinder abgefragt werden. Solche Sequenzen werden nicht als sustained shared thinking gewertet, da die Beiträge der Kinder von der Fachkraft vorgegeben sind. In mehreren Kindergärten sind solche Lehrgespräche oder stark strukturierte Sequenzen zu finden, bei denen die Fachkraft ein Kind nach dem andern in einer Nacherzählaufgabe oder einer Bildbeschreibungsaufgabe abfragt.

Die Kindergärtnerin bezieht hier die andern Kinder ein und fragt diese, ob sie K1 helfen könnten (Zeile 3). Dies ist kein Redirect, da nicht das Kind selber angeregt wird, ein anderes Kind anzusprechen. Hingegen findet sich in Zeile 6 ein Redirect mit einem erwachsenen-initiierten Vorschlag (chasch sie nomol froge). Dieser Impuls wird jedoch vom Kind nicht aufgegriffen (nicht erfolgreich), die Kindergärtnerin übernimmt die Initiative und bittet K2 um eine Wiederholung des Wortes.

\section{Diskussion und Fazit}

Wie von zahlreichen Studien belegt, hat die alltagsimmanente und adaptive Sprachförderung grosses Potenzial. Für den pädagogischen Alltag in den institutionellen Kontexten der Spielgruppe, der Kita und des Kindergartens eignen sich die fünf Strategien, im Dialog mit Kindern', ,Fragen', ,Wortschatz' ,Model- 
lieren' und ,Redirect'. Sie sind nicht an vorher festgelegte Fördereinheiten gebunden, sondern können flexibel in den unterschiedlichsten Aktivitäten und Settings eingesetzt werden. Die fünf Strategien, die für das Projekt ausgewählt worden sind, werden unterschiedlich häufig und unterschiedlich adaptiv eingesetzt; inwieweit sich Typen der Sprachförderung und qualitative Unterschiede zeigen, wird noch untersucht.

Das Fallbeispiel der Erzieherin in der Kita zeigte das gemeinsame Weiterentwickeln eines Gedankens im Dialog sehr gut auf; insgesamt werden von anderen Frühpädagoginnen nur selten längere Gespräche mit demselben Kind zu einem Thema geführt. Hier besteht ein Fortbildungsbedarf, der die Bedeutung längerer Dialoge aufzeigt. Im Beispiel des Kindergartens ist - wie auch in zahlreichen andern Kindergärten - ein Lehrgespräch zu finden, das nicht als sustained shared thinking gesehen werden kann, da für die Kinder keine eigenständigen Beiträge möglich sind. Für die Spielgruppe und für Kindergruppen mit geringen Deutschkenntnissen können die Dialoge durch Verbalisieren und durch Spielen angebahnt werden.

Als adaptive und erfolgreiche Sprachförderung mit Fragen zeigt das Fallbeispiel aus der Spielgruppe, wie zunächst eine offene Frage gestellt wird, und diese dann durch eine Alternativfrage vereinfacht wird, wenn das Kind auf die offene Frage nicht antwortet. Bei allen Frühpädagoginnen zeigt sich, dass sowohl die Prädikaterststellung wie auch W-Fragewörter sehr häufig verwendet werden. Gerade diese Frageformulierungen unterstützen die Antwort des Kindes jedoch wenig. In diesem Bereich zeigt sich die Notwendigkeit von Trainings, um das Fragenrepertoire zu erweitern und zu üben, indem unterschiedlich anforderungsreiche Fragen als Sprechimpulse dem Lernstand des Kindes entsprechend formuliert werden.

Im Bereich Wortschatz unterscheiden sich die Frühpädagoginnen vor allem darin, wie sie die neu angebotenen Wörter beschreiben, mit Gestik und Mimik zusätzlich veranschaulichen und inwiefern sie sie in einen grösseren Zusammenhang stellen. Auch die Wichtigkeit der Repetition zur Festigung neuen Wortschatzes wird noch nicht von allen anerkannt. Hier unterscheidet sich das Repertoire weniger in Bezug auf die Kontexte, die Strategien können in Spielgruppe, Kita und Kindergarten ähnlich eingesetzt werden. Adaptivität ist vor allem darin gefordert, dem Sprachstand der Kinder angepasste Wörter zu wählen, dabei nicht nur Nomen, sondern auch andere Wortarten zu berücksichtigen und im Abstrahierungsgrad der Erarbeitungsphase zu differenzieren.

Auch in Bezug auf Modellieren kann die Strategie in allen drei Kontexten in einer ähnlichen Form angewandt werden. Im Fallbeispiel der Spielgruppe wie auch im Kindergarten zeigt sich, wie die Kinder das Modell übernehmen. Der Spielgruppenleiterin mit den fremdsprachigen Kindern gelang es auch ausgesprochen gut, mit Hilfe von korrektivem Feedback den Spracherwerb zu unterstützen, zugleich den Redefluss aufrechtzuerhalten und den Beitrag des Kindes anzuerkennen. Insgesamt unterscheiden sich die Frühpädagoginnen 
stark bezüglich ihrer Einstellungen zum korrektiven Feedback, wobei einige eine Demotivierung der Kinder befürchten. Auch hier zeigt sich der Bedarf einer Weiterbildung. Videosequenzen wie das hier dargestellte Beispiel des korrektiven Feedbacks durch die Spielgruppenleiterin können dabei zielführend verwendet werden.

Redirect wird in der Regel eher wenig eingesetzt. Die hier dargestellten Fallbeispiele sind insofern repräsentativ für den geringen Einsatz dieser Sprachförderstrategie. Bei einigen wenigen Frühpädagoginnen des Samples ist ein regelmässiger Einsatz des Redirects festzustellen. Die didaktischen Formen des Kindergartens machen häufig eine selbständige Zusammenarbeit der Kinder notwendig, die eine hohe Sprachkompetenz im Aushandeln mit anderen Kindern erfordert. Diese Sprachkompetenz könnte mit Redirect angebahnt werden.

Die Fallbeispiele zeigen jedoch nicht nur viele Ähnlichkeiten zum Repertoire der Sprachförderstrategien der anderen Frühpädagoginnen, sondern auch deutlich individuelle Stile, die nicht nur durch den Kontext oder die Sprachkenntnisse der Kinder, sondern eher durch einen persönlichen Kommunikationsstil der Frühpädagogin erklärbar sind. In welcher Weise die Schwerpunkte in der Umsetzung der Sprachförderstrategien durch die individuellen professionellen Kompetenzen der jeweiligen Frühpädagogin oder durch die Bedingungen des Kontexts (Alter der Kinder, Mehrsprachigkeit, zeitliche Intensität des Angebots) begründet werden können, ist Gegenstand weiterer Untersuchungen.

Damit das Repertoire der Sprachförderstrategien erweitert werden kann, könnten Formen der Weiterbildung und des Trainings angezeigt sein, die den konkreten Kommunikationsstil der frühpädagogischen Fachkraft und den Kontext in Bezug auf die Kindergruppe berücksichtigen. Weiterbildungen können mit einer Analyse der Sprachförderkompetenz wie auch einem individuell zugeschnittenen Coaching ergänzt werden.

\section{Dank}

1 Das Forschungsprojekt Sprachförderung im Alltag wurde durch die finanzielle Unterstützung der Internationalen Bodensee Hochschule, der Stadt St. Gallen, der Schweizer Arbeitsgemeinschaft Logopädie und der Stiftung Ravensburger ermöglicht. Bei der Videokodierung arbeiteten Praktikantinnen des Master early childhood studies mit: Lena Hollenstein, Sarah Kaye, Susanne Mock Karine Müller, Monika Schwitter, Silvia Suter.

\section{Literatur}

Albers, T. (2009). Sprache und Interaktion im Kindergarten. Eine quantitativ-qualitative Analyse der sprachlichen und kommunikativen Kompetenzen von drei-bis sechsjährigen Kindern. Bad Heilbrunn: Verlag Julius Klinkhardt.

Anders, Y. (2012). Modelle professioneller Kompetenzen für frühpädagogische Fachkräfte. Aktueller Stand und ihr Bezug zur Professionalisierung. Expertise zum Gutachten "Professionalisierung in der Frühpädagogik» im Auftrag des Aktionsrats Bildung. München: vbw - Vereinigung der Bayrischen Wirtschaft e.V. Zugriff am 08.04.2014 unter http://www. bvktp.de/files/expertise_modelle_professioneller_kompetenzen.pdf 
Beck, E., Baer, M., Guldimann, T., Bischoff, S., Brühwiler, C., Müller, P., Niedermann, R., Rogalla, M. \& Vogt, F. (2008). Adaptive Lehrkompetenz. Analyse und Struktur, Veränderung und Wirkung handlungssteuernden Lehrerwissens. Münster: Waxmann.

Best, P., Laier, M., Jampert, K., Sens, A. \& Leuckefeld, K. (2011). Dialoge mit Kindern führen. Die Sprache der Kinder im dritten Lebensjahr beobachten, entdecken und anregen. Weimar: Verlag das Netz.

Buschmann, A. (2009). Heidelberger Elterntraining zur frühen Sprachförderung: Trainermanual. München: Elsevier.

Buschmann, A. \& Jooss, B. (2011). Alltagsintegrierte Sprachförderung in der Kinderkrippe. Effektivität eines sprachbasierten Interaktionstrainings für pädagogisches Fachpersonal. Verhaltenstherapie \& psychosoziale Praxis, 43(2), 303-312.

Briedigkeit, E. (2011). Institutionelle Überformung sprachlicher Herkunftsmuster. Realisation von Fragetypen im Erzieherin-Kind(er)-Diskurs. Empirische Pädagogik, 25(4), 499-517.

Dannenbauer, F. M. (1994). Zur Praxis der entwicklungsproximalen Intervention. In H. Grimm \& S. Weinert (Hrsg.), Intervention bei sprachgestörten Kindern. Voraussetzungen, Möglichkeiten und Grenzen. (S. 83-104). Stuttgart: Fischer.

Dannenbauer, F. M. (2002). Grammatik. In S. Baumgartner (Hrsg.), Sprachtherapie mit Kindern. Grundlagen und Verfahren. (S. 105-161). München: Reinhardt.

Dickinson, D. K. \& Porche, M. V. (2011). Relation between language experiences in preschool classrooms and children's kindergarten and fourth-grade language and reading abilities. Child Development, 82(3), 870-886.

Fried, L. (2008a). Professionalisierung von Erzieherinnen am Beispiel der Sprachförderkompetenz - Forschungsansätze und erste Ergebnisse. In H. von Balluseck (Hrsg.), Professionalisierung in der Frühpädagogik (S. 265-277). Opladen: Barbara Budrich.

Fried, L. (2008b). Bildung und didaktische Kompetenz. In W. Thole, H.-G. Rossbach, M. Fölling-Albers \& R. Tippelt (Hrsg.), Bildung und Kindheit. Pädagogik der Frühen Kindheit in Wissenschaft und Lehre (S. 141-151). Opladen: Barbara Budrich.

Gasteiger-Klicpera, B., Knapp, W. \& Kucharz, D. (2010). Abschlussbericht der Wissenschaftlichen Begleitung des Programms «Sag' mal was - Sprachförderung für Vorschulkinder». Pädagogische Hochschule Weingarten. Zugriff am 08.04.2014 unter http://www.sagmalwas-bw.de/media/WiBe\%201/pdf/ PH-Weingarten_Abschlussbericht_2010.pdf

Girolametto, L. Weitzman, E., Lefebvre, P. \& Greenberg, J. (2007). The Effects of In-Service Education to Promote Emergent Literacy in Child Care Centers: A Feasibility Study. Language, Speech and Hearing Services in the Schools, 38(1), 72-83.

Gretsch, P. \& Fröhlich-Gildhoff, K. (2012). Evaluation der Sprachfördermassnahmen für 3-5jährige Kinder in der Stadt Freiburg. In K. Fröhlich-Gildhoff et al. (Hrsg.), Forschung in der Frühpädagogik V. Schwerpunkt: Naturwissenschaftliche Bildung - Begegnungen mit Dingen und Phänomenen. (S. 275-304). Freiburg: FEL-Verlag.

Hopf, M. (2012). Sustained Shared Thinking im frühen naturwissenschaftlichen Lernen. Münster: Waxmann.

Huttenlocher, J., Vasilyeva, M., Waterfall, H. R. \& Vevea, J. L. (2007). The Varieties of Speech to Young Children. Developmental Psychology, 43 5), 1062-1083.

Hutterli, S. \& Vogt, F. (2014). Obligatorische Schule: Schuleintritt und erste Jahre. Zusammenstellung von Studien, Projekten und Instrumenten in den Kantonen zur Flexibilisierung und Individualisierung sowie zur Sprachförderung und Sozialisation/Integration. Bern: EDK. Zugriff am 08.04.2014 unter http://edudoc.ch/record/111988/files/schuleintritt_d.pdf

Itel, N. (2012). Filmvignetten zur Einschätzung sprachförderrelevanten Wissens. Ein Beitrag zur Professionalisierung von Frühpädagoginnen. Masterarbeit. St.Gallen/Weingarten: Pädagogische Hochschule St.Gallen. 
Jäger, D., Faust, V., Blatter, K., Schöppe, D., Artelt, C., Schneider, W. \& Stanat, P. (2012). Kompensatorische Förderung am Beispiel eines vorschulischen Trainings der phonologischen Bewusstheit. Frühe Bildung, 1(4), 202-209.

King, S., Metz, A., Kammermeyer, G. \& Roux, S. (2011). Ein sprachbezogenes Fortbildungskonzept für Erzieherinnen auf Basis situierter Lernbedingungen. Empirische Pädagogik, 25(4), 481-498.

König, A. (2007). Dialogisch-entwickelnde Interaktionsprozesse zwischen ErzieherIn und Kind(ern). Eine Videostudie aus dem Alltag des Kindergartens. Dissertation. Dortmund: Universität Dortmund, Fachbereich Erziehungswissenschaft und Soziologie.

König, A. (2009). Interaktionsprozesse zwischen ErzieherInnen und Kindern. Eine Videostudie aus dem Kindergartenalltag. Wiesbaden: VS Verlag.

Kucharz, D., Uemminghaus, M., Gasteiger-Klicpera, B. \& Knapp, W. (2009). Zielsetzung, Konzeptualisierung und Umsetzung von Sprachförderung im Kindergarten aus der Sicht von Erzieherinnen: Ergebnisse von Leitfaden-Interviews. Zeitschrift für Grundschulforschung, 2(2), 27-41.

Kuger, S., Sechtig, J. \& Anders, Y. (2012). Kompensatorische (Sprach-) Förderung. Frühe Bildung, 1(4), 181-193.

Lever, R. \& Sénéchal, M. (2011). Discussing stories: On how a dialogic reading intervention improves kindergartners' oral narrative construction. Journal of Experimental Child Psychology, 108, 1-24.

Licandro, U. \& Lüdtke, U. (2013). Peer-Interaktionen - Sprachbildung in und durch die Gruppe. Nifbe Themenheft. Osnabrück: Niedersächsisches Institut für frühkindliche Bildung und Entwicklung e.V. Zugriff am 08.04.2014 unter http://www.ifs.phil.uni-hannover.de/fileadmin/sonderpaedagogik/ AbteilungSprach-Paedagogik/Luedtke_1.pdf

Linke, A., Nussbaumer, M. \& Portmann, P. (2004). Studienbuch Linguistik. Tübingen: Niemeyer Verlag.

Lisker, A. (2011). Additive Massnahmen zur Sprachförderung im Kindergarten - Eine Bestandsaufnahme in den Bundesländern: Expertise im Auftrag des Deutschen Jugendinstituts. München: Deutsches Jugendinstitut e. V.

Meibauer, J. (2008). Pragmatik. Tübingen: Stauffenberg.

Motsch, J.-H. (2010). Kontextoptimierung. Förderung grammatischer Fähigkeiten in Therapie und Unterricht. München: Reinhardt.

Reis, H. T. \& Gable, S. L. (2000). Event-sampling and other methods for studying everyday experience. In H. T. Reis \& C. M. Judd (Eds.), Handbook of research methods in social and personality psychology (pp. 190-222). New York: Cambridge University Press.

Ritterfeld, U. (2000). Welchen und wie viel Input braucht das Kind? In H. Grimm (Hrsg.). Sprachentwicklung. Enzyklopädie der Psychologie (Band 3). (S. 403-432). Göttingen: Hogrefe.

Rossbach, H. G. \& Hasselhorn, M. (2012). Schwerpunkt: Kompensatorische Sprachförderung. Frühe Bildung, 1(4), 179-180.

Sachse, S., Budde, N., Rinker, T. \& Groth, K. (2012). Evaluation einer Sprachfördermassnahme für Vorschulkinder. Frühe Bildung, 1(4), 194-201.

Schöler, H. \& Roos, J. (2011). Die Ergebnisse des Projektes EVAS, der Evaluationsstudie zur Sprachförderung von Vorschulkindern in Heidelberger und Mannheimer Kindergärten. In Baden-Württemberg Stiftung (Hrsg), Sag' mal was - Sprachförderung für Vorschulkinder. (S. 102-112). Tübingen: Francke.

Schuele, C., Rice, M. L. \& Wilcox, K. (1995). Redirects. A strategy to increase peer interaction. Journal of Speech and Hearing Research, 38, 1319-1333.

Siraj-Blatchford, I. \& Sylva, K. (2004). Researching pedagogy in English pre-schools. British Educational Research Journal, 30(5), 713-730. 
Siraj-Blatchford, I., Sylva, K., Muttock, S., Gilden, R. \& Bell, D. (2002). Researching effective pedagogy in the early years. Research report No. 356. Norwich: Queen's Printer.

Siraj-Blatchford, I. \& Manni, L. (2008). "Would you like to tidy up now?» An analysis of adult questioning in the English Foundation Stage. Early Years, 28, 5-22.

Suchodoletz, W. von (2013). Sprech- und Sprachstörungen. Göttingen: Hogrefe.

Szagun, G., Steinbrink, C., Franik, M. \& Stumper, B. (2006). Development of vocabulary and grammar in young German-speaking children assessed with a German language development inventory. First language, 26(3), 259-280.

Torr, J. \& Scott, C. (2006). Learning 'special words'. Technical vocabulary in the talk of adults and preschoolers during shared reading. Journal of Early Childhood Research, 4(2), 153-167.

Vogt, F. (2013). Kompetenzen für eine adaptive Didaktik. In K. Zierer, (Hrsg.), Jahrbuch für Allgemeine Didaktik. (S. 196-202). Baltmannsweiler: Schneider Verlag Hohengehren.

Wasik, B. A. \& Hindman, A. H. (2011). Improving vocabulary and pre-literacy skills of at-risk treschoolers through teacher professional development. Journal of Educational Psychology, 103(2), 455-469.

Schlagworte: Alltagsintegrierte Sprachförderung, frühkindliche Bildung, Videoanalyse, professionelle Kompetenz, Sprachförderstrategien, Spracherwerb.

\section{L'encouragement des compétences langagières dans les pratiques quotidiennes à l'école maternelle, en crèche et dans des groupes de jeu. Études de cas basées sur des données vidéo}

\section{Résumé}

Les initiatives en faveur du développement langagier dans l'éducation de la petite enfance font l'objet d'attentes élevées. L'évaluation des effets de ces initiatives met en évidence le rôle central des apprentissages langagiers et, par conséquent, celui des professionnels amenés à soutenir, de manière adaptative, les enfants dans ces apprentissages langagiers. Le projet de recherche présenté ici propose une formation continue destinée aux éducateurs de la petite enfance travaillant à l'école maternelle, en crèche et dans des groupes de jeu en Allemagne et en Suisse. Cette formation est basée sur le développement de cinq stratégies. Dans cet article, la mise en place de ces stratégies d'encouragement des compétences langagières dans les pratiques de tous les jours est analysée sur la base des données vidéo.

Mots-clés: Développement langagier, éducation de la petite enfance, apprentissages langagiers, compétence professionnelle, éducateurs de la petite enfance, analyse de vidéo 


\section{La promozione delle competenze linguistiche nelle pratiche quotidiane, al nido, alla scuola materna e nei gruppi di gioco. Studi di caso basati su dati videoregistrati}

\section{Riassunto}

Le iniziative a favore dello sviluppo del linguaggio nell'educazione della prima infanzia godono di grandi aspettative. La valutazione degli effetti di tali iniziative mette in evidenza il ruolo centrale dell'apprendimento linguistico e, di conseguenza, dei professionisti che sostengono i bambini in questo percorso. Il progetto di ricerca qui presentato propone una formazione professionale continua per gli educatori della prima infanzia che lavorano nella scuola materna, all'asilo nido e nei gruppi di gioco, in Germania e in Svizzera, una formazione basata sullo sviluppo di cinque strategie. Nell'articolo, la loro attuazione - per la formazione delle competenze linguistiche nella pratica di tutti i giorni - viene analizzata sulla base della raccolta di dati videoregistrati.

Parole chiave: Sviluppo linguistico, educazione della prima infanzia, apprendimento linguistico, competenza professionale, educatori della prima infanzia, analisi video.

\section{Fostering language development in the everyday practices of kindergarten, nursery and playgroups: A Video study}

\section{Summary}

High expectations are set on initiatives fostering language development in early childhood education and care. Evaluation studies of their effects emphasise the relevance of fostering language development through everyday interactions over specific programs. As a result, professional competence related to language education adaptative to individual differences within early childhood is most important. This research project provides professional training focussing on five strategies for early childhood educators working in kindergarten, nursery and play groups in Germany and Switzerland. Based on video data, this contribution examines the implementation of the strategies in everyday practices.

Keywords: Language fostering; early childhood educators, early childhood education and care, professional competence, video study, language acquisition support strategies 
